\title{
Diane-Gabrielle Tremblay
}

[professeure, Sc. économiques et gestion, Télé-université, Université du Québec.]

(1994)

\section{'L'État et l'avenir de l'emploi"}

Un document produit en version numérique par Jean-Marie Tremblay, bénévole, professeur de sociologie au Cégep de Chicoutimi

Courriel: jmt_sociologue@,videotron.ca

Site web: http://www.uqac.ca/jmt-sociologue/

Dans le cadre de la collection: "Les classiques des sciences sociales"

Site web: http://www.uqac.ca/Classiques_des_sciences_sociales/

Une collection développée en collaboration avec la Bibliothèque

Paul-Émile-Boulet de l'Université du Québec à Chicoutimi

Site web: http://bibliotheque.uqac.uquebec.ca/index.htm 
Cette édition électronique a été réalisée par Jean-Marie Tremblay, bénévole, professeur de sociologie au Cégep de Chicoutimi à partir de :

Diane-Gabrielle Tremblay,

"L'État et l'avenir de l'emploi". Un article publié dans la revue Possibles, Montréal, vol. 18, no 3, été 1994, pp. 26-51.

http://www.teluq.uquebec.ca/interventionseconomiques

Diane-Gabrielle Tremblay est professeure de sciences économiques et gestion à la Télé-université, Université du Québec.

[Autorisation formelle de l'auteure de diffuser toutes ses publications accordée le 10 août 2004.]

8I Courriel : DGTrembl@teluq.uquebec.ca

Polices de caractères utilisée :

Pour le texte: Times, 12 points.

Pour les citations : Times 10 points.

Pour les notes de bas de page : Times, 10 points.

Édition électronique réalisée avec le traitement de textes Microsoft Word 2001 pour Macintosh.

Mise en page sur papier format

LETTRE (US letter), 8.5' $\mathrm{x} 11$ ',)

Édition complétée le 16 mai 2005 à Chicoutimi, Ville de Saguenay, province de Québec.

\section{Fait avec}

Macintosh 


\title{
Table des matières
}

\author{
Introduction
}

Un chômage chronique au Québec

Les effets de la récession des années 90 comparée à celle des années 80 La différenciation et la précarité des formes d'emploi

Le problème du Québec : un problème de développement et d'emploi

La compétitivité et la relance : comment et à quel prix?

\section{Références complémentaires}


Diane-Gabrielle Tremblay

\section{"L'État et l'avenir de l'emploi"}

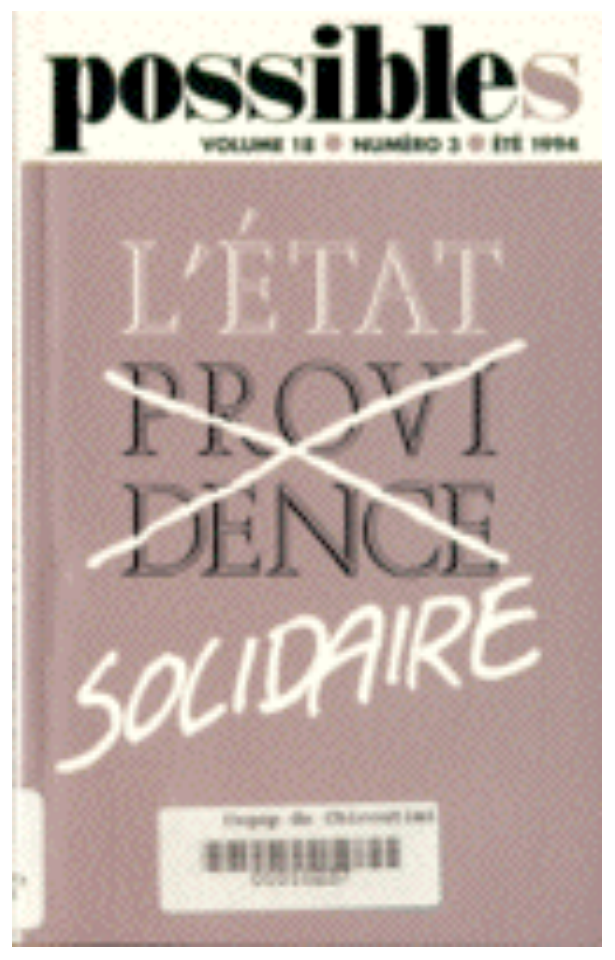

Un article publié dans la revue Possibles, Montréal, vol. 18, no 3, été 1994, pp. 26-51. 


\section{Introduction}

\section{$\underline{\text { Retour à la table des matières }}$}

Ce texte traite du rôle de l'État en regard de l'emploi ${ }^{1}$ et souligne surtout le fait que les gouvernements québécois et canadien ont généralement négligé ce rôle. De manière générale, on peut considérer qu'il existe deux grandes visions de l'État en regard de l'emploi comme en d'autres domaines d'intervention : l'État architecte, ou keynésien, qui intervient activement pour orienter ou soutenir l'activité économique, et l'État- «magicien », qui se contente de laisser-faire, espérant que des actions minimales, dispersées ici et là, permettront d'améliorer le «climat » économique et que cela suffira pour corriger les problèmes de chômage que nous connaissons. Le dernier budget fédéral du 22 février 1994 se situe tout à fait dans la lignée de cette deuxième attitude. On réduit les coûts de l'assurance-chômage et l'on espère que cette réduction de coûts suffira pour que les entreprises embauchent. On s'inspire ici de la théorie orthodoxe en économie, soit la vision néo-classique de l'économie. Or, selon la vision keynésienne ce n'est nos une baisse des coûts qui peut permettre une relance de l'économie et de l'emploi; c'est plutôt une amélioration de l'emploi qui est nécessaire pour relancer la consommation,

L'auteure tient à remercier ici la Télé-université de l'Université du Québec, tout comme le Fonds FCAR, deux organismes lui ayant fourni des fonds de recherche qui ont permis des recherches spécifiques sur l'emploi et les politiques de loi en Suède et au Canada; bien qu'il ne soit pas explicitement ou exclusivement fait référence à ces recherches ici, cellesci sont néanmoins à la base de la réflexion présentée dans ces pages. 
donc la demande pour les entreprises, puisque ce n'est qu'en présence d'une telle demande que les entreprises recommenceront à embaucher. ${ }^{2}$

De ce fait, nous considérons que l'attitude actuelle de l'État, soit de contribuer à réduire les coûts et d'inviter les entreprises à embaucher à partir de cette seule intervention, est insuffisante en regard des problèmes de chômage chronique que connaissent le Québec et le Canada. Dans les pages qui suivent, nous commencerons donc par présenter la situation actuelle de l'emploi, et surtout l'état de chômage chronique que nous connaissons, en précisant les causes de ce fait. Puis, dans une deuxième partie, nous nous interrogeons sur les solutions présentées à ce problème chronique du développement de l'emploi pour l'avenir du Québec, en traitant notamment des thèmes de la compétitivité et de la relance de notre économie.

\section{Un chômage chronique au Québec}

\section{$\underline{\text { Retour à la table des matières }}$}

Si nous faisons un petit retour en arrière, nous ne pouvons que constater que le chômage est devenu un problème chronique au Québec au cours des années 1970. Au cours des années 80 et 90, la situation du chômage n'a cessé de s'aggraver, et ce malgré une croissance assez forte à certains moments, en particulier de 1985 à 1989. En effet, malgré cette croissance, le chômage n'a cessé de progresser au Canada comme au Québec. Si l'on remonte à la fin des années 60, on constate que le chômage était déjà plus élevé au Québec que dans les autres pays de l'OCDE. Le chômage a presque doublé dans les années 70 pour atteindre 8,3\% au Canada et 10,9\% au Québec en $1978{ }^{3}$ et, depuis le début des années 80, il n'est pratiquement pas redescendu sous les $10 \%$ au Québec. Les années 90 menacent de se poursuivre sous le signe d'un toux de chômage officiel se situant à environ 12-13\% au Québec, ce qui en fait une société à chômage élevé ou chronique. En regard de la France ou du

2 Pour plus de détails sur ces théories, voir Diane-Gabrielle Tremblay, Économie du travail. Les réalités et les approches théoriques, Montréal, Éditions Saint-Martin et TéléUniversité, 1990, 544 p.

3 Les chiffres sont tirés de D.-G. Tremblay, op. cit., p. 35. 
Royaume-Uni, nous ne semblons peut-être pas si mal faire, mais en comparaison de nombre d'autres pays, comme la Norvège, la Suède, la Suisse, le Japon et l'Autriche, notre situation est loin d'être enviable. ${ }^{4}$

Cette situation de chômage élevé est certes en partie imputable à une forte augmentation de la population active, qui s'explique entre autres par le ait que les jeunes et les femmes sont de plus en plus présents sur le marché du travail; leur toux d'activité est élevé. De plus, alors que le taux d'activité des hommes diminue dans tous les groupes d'âge, celui des femmes tend à augmenter dans le groupe des 25-44 ans en particulier; il était de 71,4\% en 1987 contre 76,7\% en 1992, et la tendance historique est à la hausse. ${ }^{5}$ Cependant, il faut noter que notre pays n'est pas le seul à avoir connu une telle progression de la population active, puisqu'elle a été observée dans la plupart des pays industrialisés; il est un de ceux où cette progression est parmi les plus fortes au cours des dernières décennies, en raison de l'explosion démographique des années 50, mais cela n'explique pas entièrement notre situation de chômage.

Reconnaissons également que cette situation de chômage élevé est aussi attribuable aux récessions importantes connues au cours des années 80 et 90 . En particulier, la récession de 1990-92 a eu une incidence importante sur le chômage, puisque les pertes d'emploi se sont étalées sur une durée beaucoup plus longue que dans les années 80 et qu'un volume plus important de licenciements a été de nature permanente, par suite de nombreuses faillites et de fermetures d'entreprises.

Cependant, à notre avis, la hausse du chômage et l'incapacité de notre société à le réduire s'expliquent également par le peu d'importance accordé à l'emploi et au droit de tous à la valorisation par le travail dans notre société. Nous reviendrons sur ces thèmes plus loin.

Il convient de souligner qu'il existe aussi des disparités importantes dans les différentes régions du Québec. Ainsi, en 1992, les taux de chômage régionaux varient de 10,6\% dans la région de Québec à 21,2\% en Gaspésie. Les données historiques permettent de constater que les écarts ont toujours été importants, et que certaines régions, comme la Gaspésie, la Mauricie, les Bois-Francs, l'Abitibi-Témiscamingue et la Côte-Nord/Nord du Québec, des régions éloignées du centre, dont l'activité économique dépend souvent des

4 Nous ne traiterons pas ces pays en détail ici, mais le document de base et la série télévisée reliée au cours «Développement économique et emploi » (ECO-3005) de la Télé-Université en témoignent abondamment.

5 Voir Tremblay, op. cit., pour les définitions et des données détaillées. 
ressources naturelles et de quelques grandes entreprises, ont souvent connu des taux de chômage élevés. Bref, la situation de chômage élevé n'est pas nouvelle pour ces régions et encore, ces taux de chômage officiels sousestiment généralement la réalité du sous-emploi dans ces régions.

En effet, il existe également des écarts importants entre les taux d'activité 6 des différentes régions, ce qui signifie que des gens ont cessé de chercher du travail, découragés par la situation de chômage chronique. Ainsi, non seulement le taux de chômage est-il le plus élevé en Gaspésie, mais le taux d'activité y est le plus faible du Québec. Les régions du Bas-Saint-Laurent, du Saguenay/Lac St-Jean et de l'Abitibi-Témiscamingue (et à un moindre degré la Mauricie/Bois-Francs et l'Estrie) ont des taux 'activité nettement plus faibles que les autres régions. Les inégalités de développement se traduisent clairement dans ces indicateurs, qui montrent à quel point le marché du travail est déprimé dans certaines régions du Québec et ce, depuis plusieurs décennies.

Pourtant, depuis au moins trente ans, l'État a prétendu corriger ces disparités par des politiques et mesures diverses de développement régional. $\mathrm{Si}$ ces politiques et financements ont certes fourni des équipements et des institutions aux régions, ce qui n'est pas négligeable, il faut reconnaître que le succès n'est pas total. Encore aujourd'hui, les régions souffrent de problèmes de développement, et plusieurs d'entre elles se vident de plus en plus. ${ }^{7}$

La durée du chômage s'allonge également et cela influe sur la gravité d'une situation de chômage, en particulier pour les individus en cause. Traditionnellement, c'est l'Europe qui est généralement caractérisée par un chômage de longue durée; bien que le Québec n'ait pas rejoint l'Europe sur ce plan, on note toutefois un allongement du chômage, et donc un certain rapprochement de la situation européenne. On constate que le nombre moyen de semaines de chômage a augmenté de 19 à 22,6 au Canada et de 20,4 à 26,1 au Québec de 1986 à 1990. Il faut toutefois rappeler que le Canada est parmi les pays où le chômage de longue durée (un an et plus selon la définition de l'OCDE) est le moins élevé. Seule la Suède connaît un taux inférieur, soit $4,8 \%$, contre $5,7 \%$ pour le Canada en 1990. Cette situation apparemment

6 Rappelons que le taux d'activité représente les personnes en emploi ou à la recherche d'un emploi par rapport à l'ensemble de la population de 15 ans et plus.

7 À ce sujet, voir D.-G. Tremblay et Vincent Van Schendel, Économie du Québec et de ses régions, Montréal, 1991, Éditions Saint-Martin, 649 p. et D.-G. Tremblay et Jean-Marc Fontan, Le Développement économique local; la théorie, les pratiques, les expériences, livre associé au cours «Développement économique local » de la Télé-Université, à être publié chez un éditeur à l'été 1994. 
favorable s'explique par le fait qu'il existe un grand nombre d'emplois précaires, de courte durée, qui permettent aux personnes en chômage d'interrompre leurs périodes de chômage, ce qui diminue artificiellement la durée du chômage, mais augmente l'instabilité en emploi.

Ici encore, il faut imputer la responsabilité de cette aggravation de la durée du chômage, en partie tout au moins, au fait que l'État n'ait apparemment pas trouvé la bonne solution, ou ne soit pas intervenu suffisamment ou correctement sur ce plan. En effet, confronté à des problèmes analogues, soit la mondialisation des marchés, la délocalisation d'un grand nombre d'entreprises vers des pays à bas coûts de main-d'œuvre et ainsi de suite, d'autres pays ont mieux réussi à tirer leur épingle du jeu de l'emploi; qu'il suffise de mentionner à nouveau le Japon, la Suisse, la Norvège, la Suède et l'Allemagne notamment. Bien que ces deux derniers connaissent quelques difficultés présentement, ils ont traversé les dernières décennies avec une meilleure performance que nous sur le plan de l'emploi.

\section{Les effets de la récession des années 90 comparée à celle des années 80}

$\underline{\text { Retour à la table des matières }}$

Différents facteurs, dont une croissance faible, des investissements faibles ou effectués à l'extérieur de la région ou du pays, peuvent expliquer la faiblesse de la croissance de l'emploi dans une région ou localité donnée. Cela se traduit généralement par une augmentation du chômage. Mais un autre facteur peut contribuer à accroître les problèmes de chômage, et c'est la récession.

Une récession, puisqu'il s'agit d'un recul de la production (baisse du PIB), a évidemment toujours une incidence importante sur l'emploi et le marché du travail. Cela se traduit généralement par un ralentissement ou un recul de la croissance de l'emploi, et par une hausse du chômage et de l'exclusion d'un nombre important de personnes du marché du travail. Plus la récession se prolonge, et plus les gens se retirent carrément du marché du travail, les 
chômeurs ne recherchant plus d'emploi, parce qu'ils considèrent impossible d'en trouver dans le contexte économique existant. On les appelle alors des « travailleurs découragés » et ils sont exclus des statistiques du chômage.

Il est intéressant de se pencher sur les effets des deux dernières récessions, soit celles des années 80 et 90 , car cela nous permet de voir que la dernière récession a été particulièrement dévastatrice.

Nous nous intéresserons surtout ici à la situation canadienne, puisque ce sont des données canadiennes qui permettent de comparer les deux récessions, et en particulier d'expliquer la faiblesse de la reprise que nous connaissons. Il faut toutefois souligner que cette reprise lente, avec faible création d'emploi, touche un grand nombre de pays à l'heure actuelle. Même aux États-Unis, où la croissance économique et la reprise de l'emploi sont plus fortes qu'ici, plusieurs économistes considèrent que la reprise américaine se poursuivra à un rythme plutôt lent et irrégulier dans les années 90. Comme le note Milton Friedman, il faut se préoccuper non seulement de la progression quantitative du PIB, mais également de la qualité de cette croissance et des effets qu'elle aura sur le niveau de vie du consommateur moyen. ${ }^{8}$

Malheureusement, aux États-Unis, la hausse du PIB actuelle est essentiellement imputable à une série de nouvelles réglementations - pour la protection de l'environnement, des personnes handicapées, et ainsi de suite introduites récemment. Ces réglementations augmentent les dépenses des entreprises, mais ne favorisent que très peu la consommation privée.

Par ailleurs, tant aux États-Unis qu'au Canada et en Europe, les investissements actuels des entreprises portent en grande partie sur des équipements informatiques; $40 \%$ de la croissance économique américaine de 1992-1993 leur serait attribuable. Or, s'ils favorisent la croissance économique, ces investissements portent généralement sur des équipements plus efficaces, qui exigent moins de personnel. C'est un autre des facteurs qui contribuent à une croissance avec faible création d'emploi. Nombre d'organisations internationales s'attendent donc à ce que les problèmes de chômage et d'emploi se poursuivent dans la majorité des pays européens et au Canada au cours des années 90. Cela ne devrait pas nécessairement nous décourager, mais témoigne cependant de la gravité de la situation économique actuelle à l'échelle mondiale.

8 «La voix discordante du Nobel Milton Friedman: La reprise, si reprise il y a, ne profitera guère aux consommateurs ». Entrevue parue dans L'Espresso de Rome, traduite et publiée dans le Courrier international, no 1 de 1994, p. 13. 
Les effets de la récession ont donc été particulièrement importants au cours des années 80 et 90, parce que ces récessions ont un effet sur l'exclusion de certaines personnes ou de certains groupes du marché du travail.

Si l'on analyse les indicateurs habituels de la récession, soit les variations du PIB, on a l'impression que la récession de 1990-92 est d'une ampleur inférieure à celle de 1981-1982 au Canada. On peut alors se demander pourquoi la situation économique est toujours si désastreuse et pourquoi on compte tant d'exclus du marché du travail en 1994, alors que la reprise est amorcée depuis environ un an.

Il est vrai que les indicateurs traditionnels - soit les mesures établies à partir du sommet du cycle économique au creux de la récession - tendent à faire penser que la récession des années 90 est moins sévère que celle des années 80 . Or, si l'on y regarde de plus près, on constate qu'une différence fondamentale entre les deux récessions est la durée des difficultés économiques : au cours de la récession des années 90, le recul de l'emploi s'est étalé sur une période de 28 mois alors que dans les années 80 , cela n'avait duré que 18 mois.

Et si l'on construit de nouveaux indicateurs intégrant cet aspect de la durée, comme l'ont fait des économistes de Statistique Canada ${ }^{9}$, on obtient une toute nouvelle vision de la réalité. On constate alors que les effets de la récession de 90-92 sont beaucoup plus importants que ceux de la récession de 81-82.

La récession de 1990-92 semble a première vue moins grave que celle de 1981-82 en chiffres absolus ${ }^{10}$, mais compte tenu de la durée de la baisse d'emploi et du chômage, ses effets cumulatifs sont nettement plus importants.

En effet, plus les manifestations concrètes de la récession (recul de l'emploi et hausse du chômage) durent longtemps, plus le nombre de faillites augmente, plus les pertes d'emploi se multiplient, plus les employeurs sont enclins à licencier, et plus ils retardent leurs projets d'embauche. De ce fait,

9 P. Kuhn, G. Lemaître et G. Picot, Employment, Unemployment, Hirings and Layoffs during the 1981-82 and 1990-92 Recessions. Draft document prepared for a joint Statistics Canada/Canadian Employment Research Forum Conference on Comparing the Labour Markets During the Last Two Recessions, mars 1993. Document inédit de $50 \mathrm{p}$. fourni par les auteurs.

10 Nous ne reprendrons pas les chiffres en détail, mais ils sont donnés dans Tremblay et Fontan, op. cit., ainsi que la source précédente. 
les pertes de revenus augmentent, la consommation des ménages et les investissements des entreprises ralentissent, alors que les sommes nécessaires aux programmes de soutien du revenu augmentent, réduisant d'autant les sommes disponibles pour l'intervention de l'État dans l'économie.

Mais concrètement, quel est l'effet de cette durée plus longue de la récession des années 90 ? Premièrement, le nombre de personnes ayant perdu leur emploi a été de 25\% supérieur à celui de 1981-82, selon les estimations des économistes de Statistique Canada. ${ }^{11}$ Deuxièmement et, ce qui est plus important encore, le volume de travail ou d'emploi, mesure en nombre cumulé de «personnes-mois» de travail a été inférieur de $28 \%$ en 1990-92. Troisièmement, le volume de chômage (en «personnes-mois ») a été supérieur de $68 \%$.

Les effets sont peut-être encore plus évidents dans le secteur manufacturier. Si l'on ana se simplement l'écart entre le sommet et le creux de 'activité économique, là encore, l'écart semble négligeable. Cependant, si l'on tient compte de la durée des reculs, le portrait se modifie sensiblement. Le recul de l'emploi dans le secteur manufacturier canadien s'est prolongé pendant 41 mois à l'occasion de la récession de 1990-92, alors qu'il n'avait duré que 18 mois en 1981-82. Les pertes cumulées représentent 4,7 millions de « personnes-mois » pour la récession des années 90 , comparativement à 2,8 millions seulement pour les années 80 .

Comme la récession des années 1990 se prolonge plus longtemps que celle de 1981-82, on a de plus observé une forte hausse des licenciements permanents à partir de 1992 et cela s'est poursuivi en 1993 et 1994 . Trois facteurs permettent d'expliquer ce fait. Premièrement, la durée de la récession diminue l'espoir de voir augmenter la demande à court terme, de sorte que les entreprises réduisent leurs investissements et tendent à imposer des licenciements permanents plutôt que temporaires. Deuxièmement, cette prolongation de la récession augmente aussi le nombre de faillites d'entreprises et par conséquent les licenciements permanents. Enfin, troisièmement, les entreprises «survivantes » se restructurent, et essaient de réduire leurs coûts pour faire face à la concurrence, d'où d'autres licenciements permanents. Les deux premiers facteurs sont reliés à la durée de la récession au Canada, alors que le troisième est lié à restructuration économique mondiale, et n'est donc pas propre au Canada.

11 ibid. 
Un gouvernement s'inspirant de la vision de l'État keynésien-architecte serait intervenu pour tenter de relancer plus rapidement l'activité afin de corriger les deux Premiers effets, sur lesquels il aurait pu avoir une certaine emprise, étant entendu que le dernier, lié à la restructuration économique mondiale, dépassait le cadre de ses capacités d'intervention. Rappelons que pendant toute cette période, nos gouvernements, et la plupart des médias à leur suite, se sont contentés de tenter de nous rassurer en affirmant que la récession était finie, que le PIB avait repris sa croissance, bien que celle-ci ait été fort faible.

\section{La différenciation et la précarité des formes d'emploi}

\section{$\underline{\text { Retour à la table des matières }}$}

La précarité d'emploi est une autre réalité qui s'est développée en parallèle avec la hausse du chômage, réalité que nos gouvernements n'ont pas cherché à contrer. Au contraire, ils sont eux-mêmes, à titre d'employeur, parmi les principaux utilisateurs de contrats et de travailleurs occasionnels ou temporaires. La précarité d'emploi est évidemment liée à l'augmentation du chômage dans la mesure où plusieurs personnes acceptent un emploi précaire à défaut de trouver un emploi réguler, à plein temps. Si l'on compare la situation actuelle aux années 50 et 60 , alors que les emplois tendaient à se stabiliser sous la forme de l'« emploi régulier à plein temps », on constate que les dernières décennies ont vu se développer de nouvelles formes d'emploi, qui sont souvent caractérisées par la précarité. Le développement des activités de services, qui représentent aujourd'hui 70\% des emplois au Québec et en Amérique du Nord en général, a également contribué à la différenciation des emplois et à la précarité.

La précarité d'emploi est une réalité importante pour un grand nombre de personnes sur le marché du travail d'aujourd'hui. Comme c'est le cas pour le chômage, toutes les catégories sociales ne sont pas concernées de la même façon par les formes d'emploi différenciées ou précaires. Les hommes et les femmes sont à peu près également touchés par le chômage, mais étant donné leur forte représentation dans les services, les femmes sont parfois 
«protégées» du chômage, dans une certaine mesure, précisément parce qu'elles acceptent des emplois temporaires ou à temps partiel.

Les femmes de tous âges sont ainsi touchées par les formes d'emplois non standard, alors que ce sont surtout les jeunes hommes (15-24 ans) et, dans une moindre mesure, les hommes vieillissants (45 et plus) qui sont touchés par ces formes d'emplois. Ainsi, malgré leur plus faible représentation sur le marché du travail, les femmes constituent plus de la moitié (54\%) des personnes ayant des formes d'emploi non standard au Canada et au Québec. Les jeunes de 1524 ans (parmi lesquels on retrouve des femmes) représentent pour leur part $40 \%$ des personnes ayant des formes d'emploi atypiques. ${ }^{12}$

À l'heure actuelle, on évalue qu'entre un tiers et la moitié des travailleurs du Québec et du Canada ne travaillent pas à plein temps toute l'année et, de ce fait, ne se conforment pas à la «norme » établie en matière d'emploi ${ }^{13}$, ce qui les exclut souvent des avantages liés à un poste régulier, principalement les avantages sociaux et assurances diverses. Il est clair que la norme de l'emploi à temps plein pendant la totalité de l'année se trouve remise en question au Canada comme au Québec; en effet, cette situation ne s'applique qu'à une personne sur deux, et à peine à plus de $44 \%$ des femmes, selon les chiffres du recensement de 1991,

Des données antérieures ${ }^{14}$ nous ont permis de constater que la norme de l'emploi à plein temps toute l'année était bel et bien plus normale ou fréquente dans les années 50 et 60. Ce sont donc effectivement les années 70-80 qui marquent le changement fondamental dans les modalités d'emploi.

L'ensemble de ces constats sur la situation de l'emploi et du chômage au Québec et au Canada nous amène à conclure que non seulement y a-t-il de plus en plus divergence entre la croissance économique et la création d'emploi, qui ne progressent plus en parallèle comme auparavant, mais qu'il y a également polarisation des situations d'emploi. En effet, on observe une progression de la différenciation et de la précarisation des emplois, un maintien et dans certains cas un accroissement des écarts entre régions et groupes de population au Québec et au Canada. Dans ce contexte, dans la mesure où l'on pense qu'il conviendrait de corriger un tel état de fait, de telles disparités, qui peut intervenir en ces matières ? Nul autre que l'État n'en a

12 Ces derniers pourcentages sont tirés de l'ouvrage du Conseil économique du Canada, Tertiarisation et polarisation de l'emploi, 1991.

13 Selon les données du recensement du Canada de 1991.

14 Reproduites dans D.-G. Tremblay, (1990). 
véritablement les moyens. Des mobilisations se sont d'ailleurs manifestées au cours des dernières décennies pour inciter l'État à développer une politique de l'emploi, une politique industrielle afin de contrer le chômage chronique que nous connaissons. Mais jusqu'ici, peu de résultats concrets, si ce n'est l'inclusion de la préoccupation de l'emploi en période de campagne électorale, mais une démission rapide face à la priorité du déficit et de la dette, une fois l'élection passée. ${ }^{15}$

\section{Le problème du Québec : un problème de développement et d'emploi}

\section{$\underline{\text { Retour à la table des matières }}$}

Le problème fondamental de l'économie du Québec, c'est un problème de choix politiques et de «développement», et non un simple problème de croissance économique, comme tendent parfois à le faire penser les médias ou les politiciens.

Rappelons qu'il existe une différence fondamentale entre les notions de développement et de croissance, et celle-ci est souvent négligée. Cela conduit les politiciens et les médias a se réjouir de tout petit frémissement positif de la croissance économique, même lorsque celle-ci n'a que peu d'effet, si ce n'est aucun, sur l'emploi et sur notre bien-être, comme c'est le cas actuellement depuis près d'un an. Alors que la croissance renvoie simplement à une dimension quantitative (hausse de la production nationale, le PIB), le développement Comporte une dimension qualitative, renvoie à des préoccupations liées à la répartition des richesses, et non seulement à leur augmentation, ainsi qu'au contenu de l'augmentation de la production (PIB) ${ }^{16}$.

Ç'a été le cas pour le niveau fédéral en 1993-1994; reste à voir l'élection au Québec en 1994.

16 Plusieurs questions peuvent se poser à cet égard: s'agit-il d'une augmentation des services d'éducation, de santé, de nouveaux biens de consommation et, dans ce dernier cas, ces biens sont-ils abordables ou réservés à quelques-uns? Ou encore s'agit-il d'une production polluante, ayant des effets négatifs sur notre qualité de vie, au travail comme à l'extérieur? Voir Tremblay et Van Schendel, op. cit. pour plus de détails. 
Malheureusement, une bonne croissance économique ne nous garantit pas que la richesse sera bien répartie, que suffisamment d'emplois seront créés. En général, croissance et emploi vont de pair, comme ce fut le cas dans les années d'après-guerre, de 1945 à 1975. Mais c'est moins le cas aujourd'hui. Pourquoi?

Il semble exister des problèmes importants liés au type de croissance ou de développement que nous connaissons actuellement au Québec. En effet, comme nous l'avons noté précédemment, même si la croissance économique qui suit une récession est très forte et de longue durée, comme ce fut le cas de 1983 à 1989 au Québec, le taux de chômage peut fort bien demeurer très élevé et nombre de régions peuvent ne ressentir aucunement les effets de la reprise. Un taux de chômage dépassant souvent les $10 \%$, un taux d'activité plus faible que l'Ontario et que plusieurs pays industrialisés, une progression des formes d'emploi dites atypiques ou particulières, une hausse importante des bas salaires en particulier dans les emplois de service, voilà l'essentiel des caractéristiques de la situation de l'emploi au Québec, comme nous l'avons vu dans les pages précédentes. À l'heure actuelle, ces problèmes deviennent d'autant plus préoccupants que l'on prédit pour les années 90 une croissance moins créatrice d'emplois qu'auparavant.

La croissance avec faible création d'emploi, ou tout au moins création insuffisante pour infléchie le taux de chômage, c'est le nouveau problème de cette fin de XXe siècle. Dans les bons manuels d'économie, il est bien dit que la croissance économique peut se diviser en deux composantes : l'emploi et la productivité. On peut avoir une croissance économique comportant une faible hausse de la productivité, mais une progression de l'emploi. On peut aussi avoir une croissance alimentée par une hausse de productivité, et qui crée peu de nouveaux emplois ${ }^{17}$. Dans l'après-guerre, nous avions généralement un peu des deux, croissance et emploi. Cependant, à l'heure actuelle au Québec comme dans plusieurs pays d'Europe, l'économie est en croissance, mais emploi ne progresse pas suffisamment.

Pourquoi ? En partie parce que les entreprises ont été échaudées par la dernière récession, que la concurrence internationale est plus vive et que les entreprises cherchent à être plus compétitives, Pour ce faire, elles optent pour des investissements en équipements plus productifs et cherchent à réduire le plus possible leur frais de personnel. Ç'a été le cas vers la fin de la dernière récession. Les entreprises investissent dans des technologies et équipements qui favorisent une hausse de la productivité et permettent des économies de main-d'œuvre. Comme la demande ne progresse cependant pas suffisamment,

17 lbid. 
elles ne peuvent affecter la main-d'œuvre ainsi économisée à d'autres tâches. Une politique de l'emploi comme celle de la Suède faciliterait alors le transfert de ces personnes vers d'autres entreprises, d'autres secteurs d'activité. Mais le soutien offert au Canada est plutôt faible, et le chômage élevé rend d'autant plus difficile la mobilité des travailleurs.

Il est certain que les investissements en équipements plus performants peuvent être nécessaires et avoir un effet positif sur la compétitivité de l'économie canadienne. À court terme toutefois, si l'État n'intervient pas pour faciliter les ajustements, les déplacements, cela se traduit par un chômage élevé et 'exclusion de plusieurs personnes. Tous les pays sont confrontés à de tels changements technologiques, à des restructurations d'entreprises. Certains États interviennent activement pour faciliter, voire accélérer les restructurations, en les soutenant par une politique industrielle et une politique d'emploi. D'autres, comme le nôtre, optent plutôt pour le laisser-faire. C'est là un choix social, un choix fait essentiellement par l'État, ou les politiciens. Bien qu'il faille évidemment s'adapter aux nouvelles technologies pour demeurer «compétitifs», certains choix en matière de technologie, d'organisation du travail et de développement des compétences en entreprise peuvent permettre de maintenir, voire d'augmenter l'emploi. ${ }^{18}$

Les entreprises visent d'abord les profits, ce qui 'est pas un mal en soi, puisque ces profits représentent généralement les investissements de demain, et que ce sont ces mêmes investissements qui peuvent créer des emplois. Il faut alors espérer que la création d'emplois soit un objectif pour nos gouvernants. Or, si ceux-ci l'affirment de plus en plus au cours des derniers mois, il n'est pas certain que l'objectif de l'emploi ait la priorité en regard de celui de la réduction du déficit et de la dette. Il semble que l'emploi permette de gagner des élections, de sorte que tous les prétendants au trône y vont de leur petit couplet en faveur de l'emploi. Cependant, lorsqu'on prend les rênes de l'État, la dette et le déficit semblent reprendre leur place et éclipser largement l'emploi. Certes, il faut se préoccuper de la réduction de la dette et du déficit, mais il faut penser simultanément à l'emploi. Si nos gouvernements ne considèrent pas l'emploi comme la priorité sociale par excellence, et ils ne l'ont jamais fait, qui le fera?

Pourtant, un constat reste préoccupant en ce qui a trait à l'évolution des dernières décennies : pour un volume de production donné, de moins en moins d'emplois sont nécessaires. Et inversement, à chaque emploi est lié, en

18 Nous pensons en particulier à la Suède, à l'Allemagne et au Japon, qui ont des attitudes différentes en matière de choix technologiques et organisationnels. 
moyenne, une production plus grande au fil des ans. Pourquoi? Parce que la prductivité a augmenté. Nous sommes donc confrontés à un choix de société. Comme les profits, la croissance de la productivité est nécessaire pour la compétitivité et l'emploi. Cependant, là aussi, des options existent. Voulonsnous d'une société où un nombre croissant de personnes sont exclues de l'emploi, du statut social qui y est associé et des possibilités de développement personnel qui y sont rattachées? Voulons-nous d'une société où un pourcentage de moins en moins important de personnes travaillent, paient des impôts et assurent la survie d'un nombre grandissant d'exclus? Un choix s'imposera certes au cours des années à venir, tant sur le plan de l'emploi que sur ceux de la fiscalité et des services publics que nous souhaitons nous donner.

$\mathrm{Au}$ cours dés dernières décennies, ces problèmes et ces choix ont commence a émerger sans que nos gouvernements s'en préoccupent beaucoup. Pour leur part, ils se sont surtout attaqués de manière obsessive à l'inflation, sans aucun égard pour le chômage qu'engendraient des politiques monétaires aussi restrictives.

Nos entreprises ont cherché à rendre leurs produits plus compétitifs sur les marchés en tentant de diminuer leurs coûts de main-d'œuvre le plus possible, afin de réduire les prix de leurs produits. Cela s'est traduit par des licenciements nombreux, des rationalisations, des fermetures d'usines, et du chômage.

En Amérique du Nord, la plupart des entreprises ont une vision à court terme, une stratégie perdante à nos yeux. La baisse des coûts de main-d'œuvre constitue certes une façon de rendre des entreprises et une économie compétitives. Mais de plus en plus de spécialistes craignent que cette voie ne conduise à l'échec dans un contexte économique où les concurrents asiatiques arrivent à produire à des coûts moins élevés que nous. Nous ne pourrons jamais retourner à des niveaux de salaires aussi bas, ou alors nous serions confrontés à d'autres problèmes, des problèmes de financement des services publics et des conflits sociaux.

La recherche de profits à court terme et le désir de " passer à travers la crise » ont souvent fait oublier l'importance de la stratégie de long terme, de la planification du développement des ressources humaines afin de faire face aux prochains coups durs. De plus en plus d'économistes considèrent qu'il faut chercher une autre voie, celle que l'on fie souvent de production «à forte valeur ajoutée ». Cela signifie qu'il faut produire de la qualité et diversifier notre production pour répondre à des segments de marché particuliers, qui 
rapportent davantage, et où la concurrence n'est pas basée uniquement sur les prix, mais plutôt sur la qualité. Et pour ce faire, il faut investir dans la R \& D, ainsi que dans la formation des travailleurs.

Dans l'avenir, pour survivre à la concurrence accrue, à la diversification des demandes de consommation, à la demande de produits de plus grande qualité, l'entreprise devra pouvoir disposer d'une base d'effectifs qualifiés et stables, tout au moins ceux qui sont essentiels au processus de production. Et si elle veut mobiliser la créativité et la productivité de ses effectifs pour mieux faire face à la concurrence, elle devra à notre avis oublier totalement la stratégie de précarisation de l'emploi. La qualité et la qualification des effectifs nous paraissent tout à fait incompatibles avec la précarité d'emploi. Ce sont deux stratégies inconciliables. Pourtant, les restructurations et rationalisations en cours vont souvent dans le sens de la précarité et des réductions d'emploi.

En effet, pour le moment, hélas, l'accent semble surtout mis sur la recherche de compétitivité, et ce, dans une perspective guerrière. Les entreprises sont en guerre les unes contre les autres, à l'échelle nationale comme internationale, et elles cherchent à se donner une meilleure compétitivité, afin de gagner cette guerre. Malheureusement, la voie choisie pour se donner cette compétitivité repose souvent sur des stratégies dont les travailleurs font les frais.

À notre avis, l'entreprise « gagnante » de l'avenir ne devrait pas chercher à déstabiliser et à insécuriser son «facteur de production » le plus important, soit ses ressources humaines. Elle tentera bien sûr de maîtriser son évolution, tant en ce qui concerne ses ressources humaines que sa production ou l'éventail des services qu'elle offre. Mais l'avenir de l'entreprise réside plutôt dans une plus grande flexibilité des technologies et de l'organisation du travail, une meilleure formation et une plus grande polyvalence des effectifs.

Pour mettre en place une telle stratégie, les entreprises devront adopter des systèmes d'emploi appropriés, des systèmes d'emploi favorisant la stabilité en emploi, la formation des salariés, l'apprentissage permanent et la mobilité à l'intérieur de l'entreprise. Pour que les entreprises Puissent y arriver, notre société devra égarement modifier ses choix, les orienter en faveur de l'éducation, de la formation, de l'emploi et du développement des personnes comme de l'économie. Selon l'OCDE, l'éducation assume un rôle de plus en plus important dans la réussite économique, comme l'indiquent déjà les succès de pays comme l'Allemagne, le Japon ou la Suède. 
Les entreprises nord-américaines n'ayant pas traditionnellement favorisé cette voie, il faudrait à notre avis une intervention de l'État pour les inciter à modifier leur comportement. L'année 1994 amène de nouveaux gouvernements à Québec comme à Ottawa; il nous faut espérer que l'objectif de l'emploi ne restera pas qu'une promesse électorale, des vœux pieux rapidement oubliés une fois l'élection passée.

\section{La compétitivité et la relance : comment et à quel prix?}

\section{$\underline{\text { Retour à la table des matières }}$}

La compétitivité et la croissance économique sont certes des préoccupations de plus en plus importantes de nos gouvernants. Ces thèmes reviennent de plus en plus couramment dans les discours. Dans cette section, nous voulons nous interroger sur le sens et la portée de ce concept de compétitivité et sur le type de croissance ou de relance économique qu'il peut sous-tendre.

La mot «compétitivité » est à la mode; on l'entend partout, mais on se demande parfois ce qu'il veut dire. On se demande ce qu'est ce nouveau leitmotiv des politiciens, des économistes et de nombreux autres groupes sociaux. En principe, en économie, la compétitivité, c'est la façon dont les acteurs économiques se comportent, les entreprises et les travailleurs essentiellement, mais aussi les nations. Dans notre contexte de marchés concurrentiels, c'est l'idée que chaque acteur, surtout l'entreprise, cherche à s'assurer des avantages dit «compétitifs »: meilleur prix, meilleure qualité, etc. pour vendre son produit.

Mais aujourd'hui, la compétitivité, c'est plus qu'une façon d'être ou de se comporter dans le milieu des affaires. Le malheur, c'est que le moyen est devenu une fin en soi. Et c'est devenu une fin en soi, et non seulement pour les entreprises, mais aussi pour les États et pour les sociétés tout entières. ${ }^{19}$

19 Voir à ce sujet, l'excellent article de Ricardo Petrella, à qui nous empruntons quelques idées, parce qu'elles correspondent tout à fait aux nôtres, et qu'il les a si bien exprimées. il s'agit de «L'évangile de la compétitivité », dans Les Frontières de l'économie globale, série « Manières de voir », no 18, Paris, Le Monde diplomatique, 1993, pp. 14-15. 
Les politiciens, les financiers, les ingénieurs, tout le monde ne jure plus que par la compétitivité. Même dans l'enseignement et la formation, on ne demande plus à l'école et à l'université de développer des personnes; on leur demande de préparer un capital humain le plus performant possible pour que l'économie du pays soit plus compétitive face aux concurrents étrangers. Et c'est là le problème; comme le dit si bien Ricardo Petrella, c'est devenu un évangile! Mais c'est un évangile particulier. Ici, pas de place pour la compassion ou des sentiments du genre. L'évangile de la compétitivité se définit en termes guerriers.

Nous sommes engagés dans une guerre technologique, économique et industrielle sans merci à 'échelle mondiale. Chaque État cherche à devenir plus compétitif que son voisin pour ne pas se faire tuer, éliminer de la carte.

C'est là une nouvelle religion dominée par quelques dizaines de grands évangélistes de renommée internationale, comme Michael Porter par exemple, ou d'autres grands prêtres américains lui ont prétendu aller enseigner leur art à la nouvelle Russie et à l'Europe de l'Est, avec le succès que l'on connaît... Les consultants en management et en gestion sont la catégorie la mieux formée en la matière, et la plus convaincante... Mais il y a aussi beaucoup de journalistes qui ne connaissent pas bien les théories économiques et qui se laissent facilement emporter par cette vision un peu simpliste des choses. Plusieurs d'entre eux accordent beaucoup trop d'importance au « World competitiveness index » produit par une institution suisse. Cet ouvrage véhicule une vision plutôt réductrice de l'économie mondiale : on classe les États en fonction d'indicateurs limités et ces États sont comme des armées qui s'affrontent sur le champ de bataille.

Les États doivent inciter leurs armées - leurs entreprises - a innover, faire de la recherche-développement, de la spéculation, du dumping, tous les moyens sont bons pour vaincre. On s'en doute, il y aura évidemment peu de gagnants et certains États, et des continents entiers (l'Afrique en particulier), seront exclus.

Mais la compétitivité, c'est comme la grâce : on l'a ou on l'a pas. Les nations qui l'ont survivront; celles qui ne l'ont pas en mourront... Cela veut dire qu'il y aura beaucoup de monde d'exclu_

Tous les États semblent soumis à «l'évangile » de la compétitivité et les conséquences peuvent être plus désastreuses pour certains États que pour d'autres. Nous pensons en particulier aux pays de l'Afrique, totalement 
marginalisés, détruits ou exclus du monde par l'application de ces dogmes par le FMI ou la Banque mondiale. Mais certains États d'Amérique centrale, d'Amérique du Sud ou d'Asie seront sans doute également exclus, ou tout au moins connaîtront des difficultés.

Cela ne veut pas dire que le Québec et le Canada ne doivent plus chercher à innover, à faire de la recherche-développement, a conquérir des marchés... Il ne s'agit pas de nous asseoir sur le champ de bataille et de nous faire écraser par les États- «armées » ennemis.

Nous ne disons pas que nous ne devons pas former nos ressources humaines, améliorer la qualité de nos produits et innover pour développer notre économie... et surtout nos emplois. Au contraire, d'autant plus que nous avons des retards à rattraper sur ce plan. Cependant, la compétitivité ne doit pas être un but en soi.

À nos yeux, les objectifs de l'activité économique, ce sont plutôt le développement social, culturel, technologique, politique et économique de l'humanité, des individus et non seulement des entreprises ou des Étatsnotions. Dans nos livres d'économie, figure aussi l'objectif d'assurer une répartition équitable des richesses et le développement des personnes qui se trouvent sur le territoire national.

Tout cela disparaît lorsque la compétitivité devient l'objectif unique et que l'on oublie les quelques millions de personnes exclues de l'emploi Jans les pays développés, sans compter les quelques milliards de personnes qui ne vivent pas dans les pays développés et qui seront effacées de la surface du globe si les pays développés ne se préoccupent que de compétitivité au sens strict. En effet, la compétitivité c'est fondamentalement un jeu à trois, entre l'Amérique du Nord, le Japon et l'Europe (de l'Ouest

Or, nous pensons que les défis d'aujourd'hui dépassent ces trois lieux et nous concernent directement. Nous ne pourrons pas effacer la crise économique et les problèmes nucléaires de l'ex-URSS, les problèmes environnementaux du Sud, les migrations internationales du Sud vers le Nord et tant d'autres problèmes, en nous préoccupant uniquement de compétitivité. Cela exige une coopération qui passe par la recherche et le développement socio-économique de ces régions.

Il ne faut pas se faire d'illusions. Si nous ne les aidons pas et si nous continuons de jouer à la guerre entre pays développés, nous risquons d'être confrontés à des problèmes bien plus importants plus tard... 
Il faut voir plus grand, surtout dans le contexte actuel de mondialisation que nous connaissons. À cet égard, sans doute convient-il de conclure cet article sur d'autres perspectives de relance économique plus favorables à l'emploi et au développement socio-économique de l'ensemble de la planète. Dans une édition du Washington Post, l'économiste Robert Kuttner affirmait qu'il fallait repenser notre vision économique et organiser un sommet des pays industrialisés en vue d'une relance de l'emploi à l'échelle mondiale ${ }^{20}$. Nous sommes tout à fait en accord avec lui et c'est pourquoi nous reprendrons ici les quatre ingrédients de sa recette. Celle-ci dépasse le cadre strict d'un Étatnotion, mais témoigne bien du fait que dans un contexte de mondialisation, ce sont aussi à ces enjeux globaux que les États devraient s'intéresser pour améliorer la situation socio-économique et celle de l'emploi de leurs commettants. En effet, sans doute les politiques de l'emploi et les politiques industrielles seraient-elles plus efficaces dans un contexte où il y aurait plus grande concertation internationale des États.

La première proposition de Kuttner en vue d'une relance de l'économie mondiale appelle à des dépenses publiques plus importantes afin de remplacer les dépenses militaires de la guerre froide, qui étaient le moteur de la croissance économique. Compte tenu des coupures effectuées au budget de la défense du Canada, coupures qui paraissent fort justifiées, cette proposition est d'autant plus pertinente pour nous.

Kuttner affirme aussi qu'il faut un programme plus important de crédits en faveur de l'ancien bloc des pays de l'Est, un programme qui serait comparable à la reconstruction de l'après-guerre. Cette proposition s'inspire, bien sûr, de préoccupations humanitaires que l'on comprend bien si l'on a visité quelques régions de l'ancien bloc de l'Est, mais du point de vue économique, elle peut également fournir de nouveaux marchés pour les exportations nordaméricaines.

En troisième lieu, Kuttner propose des négociations internationales pour normaliser conditions de travail et salaires dans les pays concurrents, au Sud comme au Nord. La conclusion de l'Accord de libre-échange entre le Canada, les États-Unis et le Mexique rend cette proposition particulièrement pertinente pour nous, et l'unification de l'Europe également. En effet, si un travailleurconsommateur canadien peut profiter des faibles coûts de main-d'œuvre du Sud par l'importation de produits à bas pris, il sera moins heureux si son salaire diminue en raison de cette concurrence ou s'il perd son emploi à cause

20 Voir Ricardo Petrella, op, cit. 
de ces importations. Les pays en voie de développement devraient offrir de meilleures conditions de travail et mieux rémunérer leurs travailleurs; sinon, les salaires mondiaux vont continuer de s'aligner à la baisse... et il faudra attendre longtemps une relance de l'emploi !

Enfin, une dernière proposition a trait à la tenue de négociations commerciales, afin d'éliminer les protectionnismes, et à la mise en place d'une agence internationale capable de faire respecter règles du jeu. Le deuxième aspect de cette proposition est particulièrement important pour les États-Unis, qui se posent souvent en «gendarme », à la fois juge et partie dans nombre de conflits commerciaux, en particulier avec le Canada.

Toutes ces propositions ne réduisent pas la pertinence d'une politique d'emploi et d'une politique industrielle cohérente, qui relèvent de la responsabilité de l'État québécois et canadien, mais s'ajoutent à leurs responsabilités en matière d'emploi, compte tenu du contexte de la mondialisation que nous ne pouvons plus ignorer.

\section{Références complémentaires}

\section{$\underline{\text { Retour à la table des matières }}$}

Pour compléter la lecture de cet article, on pourra consulter les ouvrages suivants :

D.-G. Tremblay, L'emploi en devenir, Québec, Institut québécois de recherche sur la culture, collection Diagnostic, 1990.

D.-G. Tremblay, «La formation dans les entreprises : réalités et défis », revue Possibles, automne 1992, Montréal.

D.-G. Tremblay, «Les tendances de l'emploi : flexibilité et précarité », dans D.-G. Tremblay, sous la direction de, Travail et société. Une introduction à la sociologie du travail, Montréal, Éditions de l'Agence d'Arc, 1992, 645 p. (article : pp. 457-500). 
D.-G. Tremblay, «Innovation et marchés internes du travail dans le secteur bancaire; vers un modèle multidimensionnel de l'innovation ", Technologies de l'information et société, vol. 4, no 3, Paris, Dunod, 1992, p. 351-380.

D.-G. Tremblay et Gilles Gateau, «Les programmes gouvernementaux pour les jeunes : une nouvelle orme de gestion de non-emploi ? L'exemple des TUC et des travaux communautaires en France et au Québec », dans Interventions économiques (1988), Flexibilité du travail, no 19, Montréal, Éditions Saint-Martin, 1988.

Fin du texte 